Killer of Prune in Drosophila melanogaster ${ }^{9,10}$. The effects of the $n m 23-H 1$ gene in tumour progression and metastasis probably depend on both its level of expression and the occurrence of specific mutations in its protein.

Christina L. Chang, Xlao-xiang Zhu,

Didier H. Thoraval, David Ungar,

Jawhar Rawwas, Nlvedita Hora,

John R. Strahler, Samir M. Hanash

Department of Pediatrics,

Eric Radany

Department of Radiation Oncology, University of Michigan School of Medicine, R4451 Kresge I, Box 0510 ,

Ann Arbor,

Michigan 48109, USA

\section{Retinal cone differentiation}

SIR - During retinal development, differentiation of each cell type follows a strict timetable: in rodent retinae $e^{1,2}$ the short-wave-sensitive cone photoreceptor molecule (S pigment) is expressed before the middle-wave-sensitive $(\mathrm{M})$ cone pigment, and we now show that this appearance of $\mathrm{M}$ cones after the $\mathrm{S}$ cones is a result of their development from $\mathrm{S}$ cones. So S-pigment expression must be the default pathway in cone differentiation, with prospective $\mathrm{M}$ cones switching to the green-sensitive pathway only secondarily in response to an as yet unknown factor.

We followed the kinetics of expression of $\mathrm{S}$ and $\mathrm{M}$ cones in the developing rat and gerbil retina with OS-2 and COS-1, antibodies specific for the carboxyl terminus of the $\mathrm{S}$ and $\mathrm{M}$ pigments, respectively ${ }^{3}$. In the rat, immunopositive $S$ cones started to appear on the fifth postnatal day (P5) and their density increased until P11; the first COS-1-positive $\mathrm{M}$ cones emerged at $\mathrm{P} 9$, and reached the maximum density at P15 (see $a$ in figure). The number of the OS-2-positive cones then dropped by almost 95 per cent, whereas COS-1positive cones decreased only slightly.

This sudden drop in the S-cone forerunners coinciding with the accumulation of $\mathrm{M}$ cones indicated that the $\mathrm{S}$ cones might be changing phenotype and transforming into $M$ codes rather than dying out. Although programmed cell death probably influences adult patterns, we saw no degenerating or dying cones during the critical period of cone development, making this an unlikely explanation for the fall in $\mathrm{S}$ cones. Neither can it be accounted for by the roughly ten per cent expansion of the retinal surface between P10 and P30, nor by the lateral movement of cones during morphogenesis, as both cone types are uniformly distributed across the entire retina at all ages.

Owing to the rapid randomization of newly synthesized outer-segment proteins

$a$
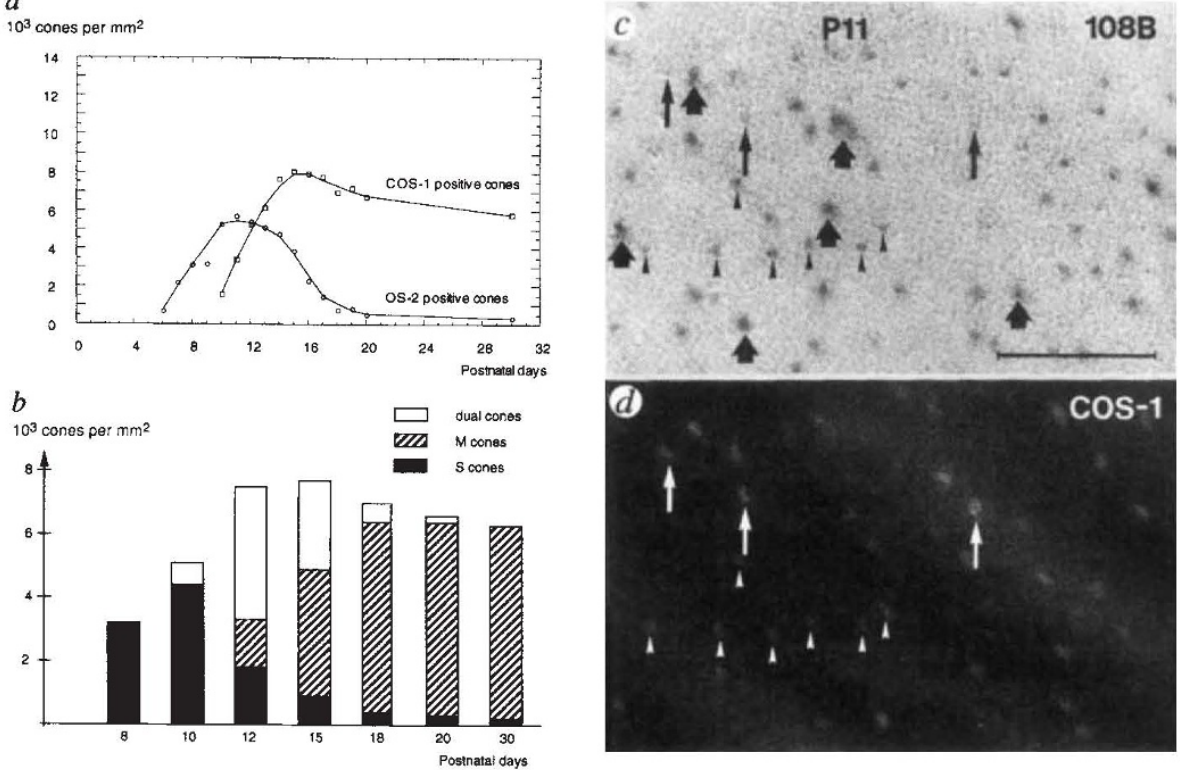

a, Quantitative analysis of cone development in rat retinae. OS-2- and COS-1-positive cones were counted on unit $\left(0.1 \mathrm{~mm}^{2}\right)$ areas and plotted as a function of postnatal time. The bound antibodies were detected with rhodamine and fluorescein isothiocyanate (TRITC and FITC, respectively). Topographically identical areas, located halfway between the optic nerve head and the superior edge of the ora serrata, were selected for comparison. Mean values derived from three animals of the same age were used. Cones expressing S pigment appear earlier (OS-2, circles), but after reaching their peak, the curve drops steeply. M-pigment-containing cones emerge later (COS-1, squares), and after peaking, the curve declines only slightly to plateau just below the maximum. $b$, Diagram showing the densities of cones stained with either 108B, a rabbit polyclonal antibody raised against a C-terminal epitope of the human blue visual pigment ${ }^{6}$ (S cones), or COS-1 (M cones), or both (dual cones). Bound antibodies were detected with FITC and TRITC, respectively, on doubie-labelled rat retinae. Note that dual cones arise during only a short period of the development. Samples shown are from identical areas of individual retinae, but the entire retina contained the same distribution of differently stained cones at each age. $c, d$, Double immunolabel of an 11-day-old rat retina with 108B and COS-1. Bound antibodies were detected by diaminobenzidine (108B) and FITC (COS-1). Pairs of photographs were taken from the same retinal area using Nomarski optics $(c)$ and a fluorescent filter $(d)$. Cones are mostly stained with both antibodies (arrowheads). There are a few heavily labelled $108 \mathrm{~B}$ cones (genuine $\mathrm{S}$ cones) that are not recognized by COS-1 at all (thick arrows). Cones strongly labelled by COS-1 generally bind 108B only weakly (thin arrows). Scale bar, $25 \mu \mathrm{m}$.

and the much slower shedding of the apical disks ${ }^{4,5}$, cones containing both pigments should be evident during the proposed shift. We used double-label immunocytochemistry to test for such cones in the rat and gerbil, by reacting whole retinae with $108 \mathrm{~B}$, an antibody against human blue opsin ${ }^{6}$, and with the M-conespecific COS-1; bound antibodies were detected using different fluorochromes or with diaminobenzidine and fluorochrome. We also reacted consecutive tangential sections alternately with OS-2 and COS-1 to identify with both pigments (not shown). OS-2- and 108B-labelled photoreceptors are known to be identical ${ }^{7}$.

Between P9 and P20, many cones bound both antibodies ( $b$ in figure), indicating the temporary coexistence of both pigments. Initially, all cones were recognized exclusively by S-pigmentspecific antibodies. Later, the majority of cones were seen to be at various stages of transition between the two phenotypes ( $c$ and $d$ ). By P30 most of these cones were undetected by $108 \mathrm{~B}$ and OS-2 and only a few, genuine $S$ cones retained the original phenotype.

Our results show that most of the earlymaturing $\mathrm{S}$ cones in the rat and gerbil retina change their phenotype to become $\mathrm{M}$ cones. The green-sensitive cones thus derive from the transforming cones, while a few cones do not undergo the shift and constitute the definitive S-cone population. The differentiation around the second postnatal week indicates that instead of colour-specificities being predetermined, the ultimate cone phenotypes will be defined only after the onset of visual pigment synthesis.

\section{Ágoston Szél*}

Theo van Veen

Department of Zoology,

University of Gothenburg,

Gothenburg, S-41390,

\section{Sweden}

\section{Pál Röhlich}

Laboratory of Electron Microscopy,

Semme/weis University Medical School, Budapest, H-1450, Hungary

* At both addresses.

1. Wang, Y. etal. Neuron 9, 429-440 (1992)

2. Szél, Á., Röhlich, P.. Mieziewska, M., Aguirre, G. \& vanVeen T I Comp Neurol 331 564-577 (1993)

Röhlich, P.\& Szél, A. Curr. Eye Res, 12, 935-944 (1993)

4. Young, R. W. Invest. Ophthal. 15, 700-725(1976)

5. Bok, D. Invest. Ophthal. vis. Sci. 26, 1659-1694 (1985).

6. Lerea, C. L., Bunt-Milam, A. H. \& Hurley, J. B. Neuron 3 367-376 (1989).

7. Szél, Á. et al. J. comp. Neurol. 325, 327-342 (1992). 